\title{
2-D real-time quantitative microwave imaging of reinforcement bars: simulations and experiments
}

\author{
J. De Zaeytijd * $\quad$ T.Maes ${ }^{*} \quad$ A. Franchois ${ }^{\dagger}$
}

\begin{abstract}
This paper treats the non-destructive localisation of reinforcement bars. It introduces a quantitative algorithm using a Gauss-Newton-type optimization to determine the radius and position of one reinforcement bar in a homogeneous medium from data collected with a bi-static linear measurement setup. The use of the Linear Sampling method as a preprocessing step and a fast analytical 2D model for the direct problem results in a robust and quasi-realtime microwave imaging system. Reconstructions from both simulated and experimental data are presented.
\end{abstract}

\section{Introduction}

For repair and renovation of concrete structures, information on the location and geometry of the reinforcement bars is a necessity. The position of the rebars is important since most processes of corrosion are directly related to an insufficient concrete cover or incorrect placement of the reinforcement. The diameter of the bars determines the bearing capacity of concrete elements. There exists a strong interest in non-destructive techniques to determine these important parameters. Recently a near-field Synthetic Aperture Radar (SAR) technique was developed in our department [1]. This technique, however, produces only qualitative images, that allow for the detection and localisation of the rebars, but with a limited accuracy in terms of position, and with almost no information about the diameter of the bars. Moreover, image artefacts are often present. Its main advantage is its speed, which makes it suited for real-time applications. In this paper we present a quantitative microwave inversion method for the detection of metal bars in free space. This is the first step towards a completely quantitative imaging algorithm for rebars in concrete. The data are collected with a bi-static linear measurement setup which measures the scattered field in reflection only. For the inversion, a GaussNewton minimization algorithm [2] is applied to a least squares datafit cost function. The evaluation of this cost function and its derivatives is very efficient due to the use of an analytical forward model that explicitely uses the facts that the bars have a

\begin{tabular}{|c|c|c|c|}
\hline *INTEC, & Ghent & University & Sin \\
\hline $\begin{array}{l}\text { ietersnieuwstraat } \\
\text { urgen.dezaeytijd }\end{array}$ & $\begin{array}{l}41, \quad 9000 \\
\text { intec.uge }\end{array}$ & $\begin{array}{l}\text { thent, Belgium, } \\
\text { be }\end{array}$ & \\
\hline $\begin{array}{l}\text { †INTEC-IMEC, } \\
\text { ietersnieuwstraat }\end{array}$ & Ghent & $\begin{array}{l}\text { University, } \\
\text {, Belgium }\end{array}$ & - \\
\hline
\end{tabular}

circular cross-section and can be modeled as perfectly electrically conducting (PEC) at microwave frequencies. To avoid the pitfall of multiple local minima of the cost function, the Linear Sampling Method (LSM) $[3,4]$ is used as a preprocessing step that finds a good initial estimate for the optimization algorithm. The resulting method is robust and provides quasi-realtime reconstructions of the reinforcement bars. It is validated on both simulated and measured scattering data.

\section{Theory and algorithm}

\subsection{Direct problem}

We consider a Perfectly Electrically Conducting (PEC) cylinder with circulair cross section placed in a homogeneous background medium. The geometry of sources and scatterer is assumed to be z-invariant, so a 2D approximation can be used. This assumption is inspired by the vertical position of the reinforcement bar and the use of only TMexcitations in the measurements. Scattering by this simple configuration can be treated analytically [5] allowing for a fast simulation of the scattered field and its derivatives.

The major difficulty is presented by the linear measurement setup, which requires the incorporation of the antenna pattern in the calculations, because the scatterer is not always in the main lobe of the antenna. Without incorporation of the antenna pattern in the direct problem, the simulated scattered field will never match the measured data. After experimental determination of amplitude and phase of the pattern it can be incorporated in the analytical calculations.

\subsection{Inverse problem}

It is known that the inverse scattering problem is ill-posed, i.e. the existence, uniqueness and stability of the solution are not simultaneously guaranteed. To address the nonexistence, caused by noise and modeling errors, the solution is redefined as the minimizer of a cost function. A least squares cost function is used:

$$
\left.Q\left(x_{0}, y_{0}, a\right)=\| \boldsymbol{E}\left(x_{0}, y_{0}, a\right)-\boldsymbol{E}^{m}\right) \|^{2}
$$


where $\boldsymbol{E}\left(x_{0}, y_{0}, a\right)$ is a vector that contains the simulated field scattered from a bar (position $\left(x_{0}, y_{0}\right)$ of the center, radius $a$ ) and $\boldsymbol{E}^{m}$ contains the measured field data. This cost function proved to have multiple local minima which makes it difficult for a local optimization method to converge succesfully.

\subsubsection{Linear Sampling}

A good initial guess can avoid the problem of multiple minima. To this end the Linear Sampling Method (LSM) is applied to the measured data $[3,4]$. The LSM solves a linear ill-posed problem to determine the support of the scatterer. To handle the ill-posedness of the linear problem a Tichonov regularization is employed. The image acquired by the method is then used to estimate the position and radius of the rebar.

\subsubsection{Gauss-Newton method}

Subsequently the Gauss-Newton method is used to find the minimizer of the costfunction (1). This local method will converge to the correct global minimum if it is started from the initial LSM estimate. The Gauss-Newton algorithm adjusts the vector of reconstruction variables $\boldsymbol{u}=\left[x_{0}, y_{0}, a\right]$ in each iteration with the update $\Delta \boldsymbol{u}=\left[\Delta x_{0}, \Delta y_{0}, \Delta a\right]$ calculated as:

$$
\Delta \boldsymbol{u}=-\Re\left[\boldsymbol{J}^{H} \boldsymbol{J}\right]^{-1} \Re\left[\boldsymbol{J}^{H} \Delta \boldsymbol{E}\right]
$$

with $\boldsymbol{J}$ the Jacobian matrix of the scattered fields $\boldsymbol{E}$ and $\Delta \boldsymbol{E}$ the vector with $\Delta \boldsymbol{E}=\boldsymbol{E}-\boldsymbol{E}^{m}$. In practice we have found it advisable to perform the optimization in two steps: first the radius is kept fixed at the LSM value, while the $x_{0}$ - and $y_{0}$-values are updated and in a second step all parameters are updated simultaneously. This seems to prevent problems with negative values for the radius of the bar and avoids the use of a constrained optimization.

\section{Experimental setup}

To perform the measurements a linear, fully automated positioning system is used. Attached on two separate rails the transmitting and receiving antennas move along the $\mathrm{x}$-axis. The antennas are positioned at different heights to prevent collision during movement. In this setup exponentially tapered horn antennas are used in the frequency range of 9-11Ghz. After calibration of the antenna-cables and alignment of the antennas, a rebar is placed in front of the antennas. Two data sets are collected, one without rebar (free space measurement) and one with the bar in place (total field measurement). For every measurement, the transmitter is

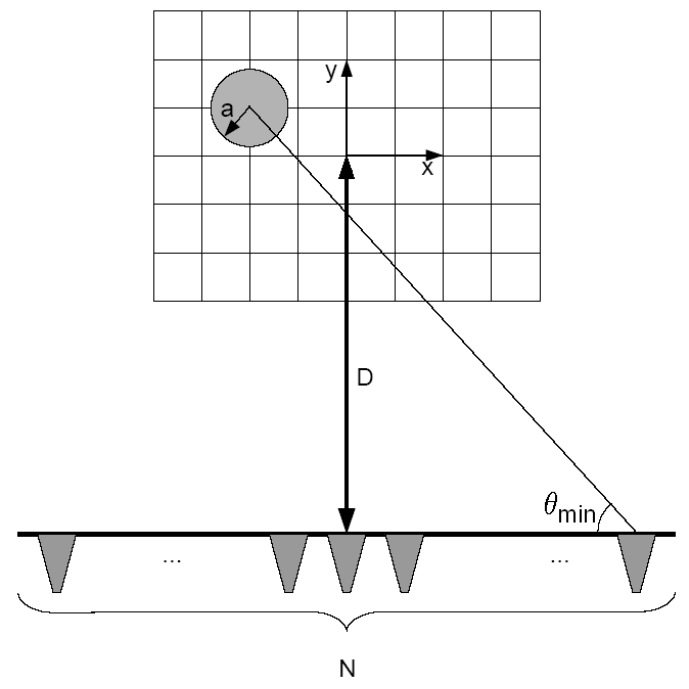

Figure 1: Overview of simulation parameters

successively placed in $N$ equidistant positions and for each transmitter position, the scattered field is measured in the same $N$ points by the receiver (see Figure 1).

\section{Calibration}

In theory the scattered field is obtained by subtracting the free space data from the total field data. Due to a variety of factors the measurements are not completely reproducible and the crosstalk between transmitter and receivers is not entirely eliminated. A rigorous calibration method utilizing known theoretical properties of the scattered field is applied on the data. This method removes the crosstalk peaks without any a priori information on the bar. The key problem still remaining is normalizing the measurements to the level of the simulated values. Due to a lack of reproducibility a method using a reference measurement cannot be used at this time.

\section{Results}

\subsection{Simulated data}

To test the performance of the proposed method, we first conducted a parameter study for following parameters: the position of the bar $\left(x_{0}, y_{0}\right)$ on a test grid, the distance $D$ between the antenna array and the center of this grid, the radius $a$ of the bar and the number of antenna-positions $N$ (see Figure 1). The antennas were always spaced $2 \mathrm{~cm}$ apart. The simulations were performed for omnidirectional antennas (line sources) and where corrupted with Gaussian noise, corresponding to a 
Signal to Noise Ration (SNR) of $50 \mathrm{~dB}$. The reconstruction error is defined as $\left\|\mathbf{u}-\mathbf{u}_{0}\right\|$, where $\boldsymbol{u}$ and $\mathbf{u}_{0}$ are the reconstructed and actual parameter vectors respectively. It was found that the method does not converge optimally in certain situations. One factor is the influence of the minimal angle $\theta_{\text {min }}$ under which the bar is seen from the antennas (Figure 1). This angle should be smaller then approximately 60 degrees to keep enough depth resolution in the measurements. This can be achieved by increasing $N$ or decreasing $D$. It was further shown that the Linear Sampling grid has to include the rebar completely. Placing the bar at the edges of the grid results in faulty intial estimates and can lead to convergence in a wrong local minimum. The mean error over all test cases that comply with the restrictions above was only $0.07 \mathrm{~mm}$. The mean execution time was $13 \mathrm{~s}$ (on a $2.4 \mathrm{Ghz}$ processor) and the mean number of iterations was 7 .

To investigate the influence of measurement noise, the following two test cases were selected : $N=51, D=0.8 \mathrm{~m}, a=6 \mathrm{~mm}$, position $1=$ $(0,0)$ and position $2=(0.15,0.05)$. The SNR values were varied from 15 to $50 \mathrm{~dB}$. Figure 2 shows the reconstruction error as a function of the SNR. In the worst case scenario (test case $1, \mathrm{SNR}=15$ $\mathrm{dB}$ ) we found an error $=0.5 \mathrm{~mm}, 7$ iterations and an execution time of $16 \mathrm{~s}$. It can be concluded that high noise levels do not significantly affect the minimizer of the cost function (although the minimal value is altered) and that the LSM can handle high noise levels.

To investigate the influence of the antenna pattern on the performance of the algorithm, the same two test cases were selected and again the reconstruction error was calculated for different SNR values (Figure 3). By adding the pattern, execution time rises drastically due to a more complex analytical calculation. The pattern narrows the measurement angle and by doing so lowers the depth resolution. The maximum error (test case 2 , SNR $=15 \mathrm{~dB}$ ) is $5.8 \mathrm{~mm}$ with 41 iterations and execution time of $206 \mathrm{~s}$, but generally, for SNR values larger than $20 \mathrm{~dB}$, it can be concluded that the algorithm yields mm-precision and has execution times in the order of minutes.

\subsection{Experimental data}

The algorithm was also applied to real measurements taken with our setup. After calibration we were able to succesfully find a solution. The rebar with radius $a=6 \mathrm{~mm}$ was placed in the vincinity of the origin $(0,0)$ of the grid at $D=1 \mathrm{~m}$ and with $N=51$. The algorithm found a position of $(-0.0007,0.0097)$ and a radius of $6.9 \mathrm{~mm}$. The es-

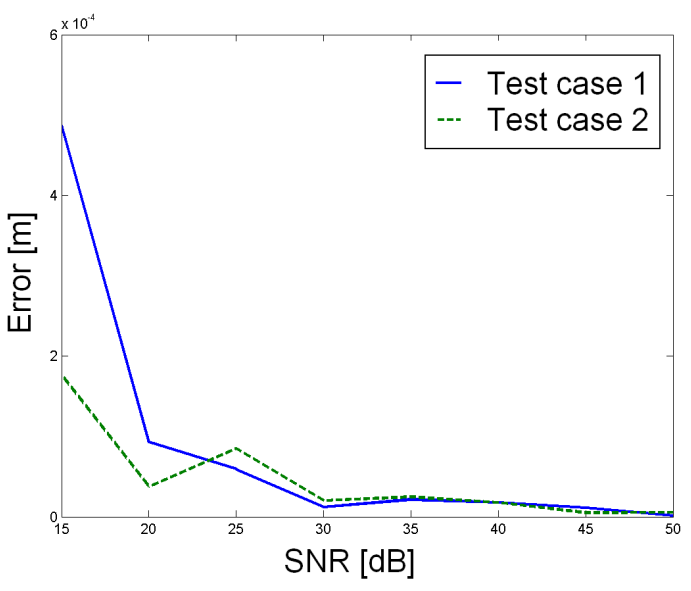

Figure 2: Reconstruction error vs. SNR without radiation pattern.

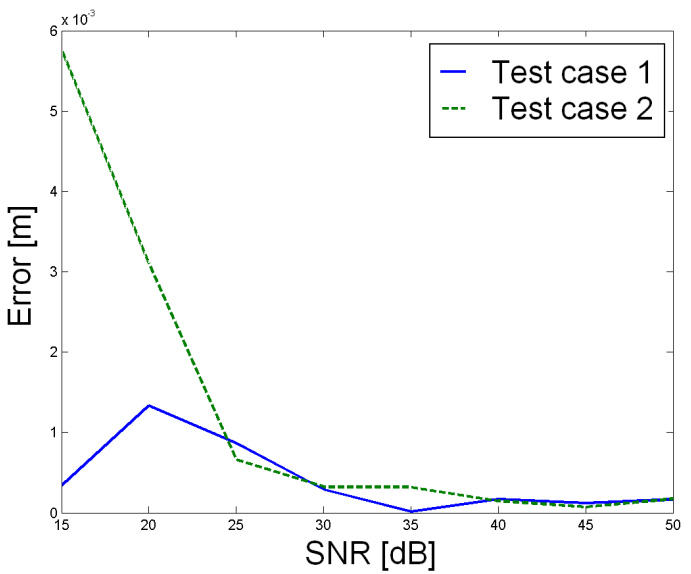

Figure 3: Reconstruction error vs. SNR with radiation pattern.

timated SNR of the measured data was $15 \mathrm{~dB}$. Since the bar was positioned manually without great precision, especially in the y-direction, this result can be called satisfactory.

\section{Conclusions}

To solve the $2 \mathrm{D}$ inverse scattering problem of finding a reinforcement bar in a homogeneous space, an algorithm, consisting of a Linear Sampling preprocessing step and an iterative Gauss-Newton optimization, was developed. A parameter study showed that mm-precision can be attained for a wide range of configurations with execution times in the order of minutes. It has also been shown that the method can be applied to experimental data with good outcome. 


\section{Acknowledgments}

Jürgen De Zaeytijd is a Research Assistant of the Fund for Scientific Research - Flanders.

\section{References}

[1] A. Franchois, P. Lewyllie, L. Taerwe, "2-D nearfield SAR for non-destructive testing of rebars in a concrete wall", Int. J. Appl. Electrom. Mech., Vol. 19, No. 1-4, pp. 333-338, 2004

[2] J. E. Dennis Jr., Robert B. Schnabel, Numerical methods for unconstrained optimization and nonlinear equations, Prentice Hall, New Jersey, 1983

[3] D. Colton, H. Haddar, M. Piana, "The linear sampling method in inverse electromagnetic scattering theory", Inverse Problems, Vol. 19, No. 6, pp. S105-S137, 2003

[4] R. Aramini, M. Brinone, M. Piana, "Applications of a No-Sampling approach to the Linear Sampling Method", Proceedings of ACES 2007, Verona, Italy, pp. 1947 - 1954, 2007

[5] J. Van Bladel, Electromagnetic fields, SpringerVerlag, New York, 1985 

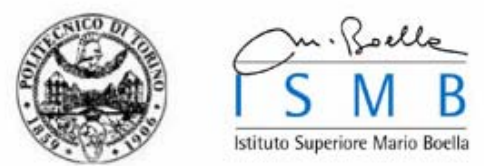

torinowireless

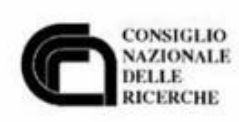

\section{- IEEE}
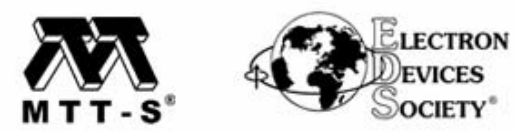

A

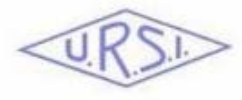

\section{International Conference on} Electromagnetics in Advanced Applications

\section{ICEAA '07}

$10^{\text {th }}$ Edition 